Document downloaded from:

http://hdl.handle.net/10251/67895

This paper must be cited as:

López Gresa, MP.; Lisón Párraga, MP.; Kim, HK.; Choi, YH.; Verpoorte, R.; Rodrigo Bravo, I.; Conejero Tomás, V.... (2012). Metabolic fingerprinting of Tomato Mosaic Virus infected Solanum lycopersicum. Journal of Plant Physiology. 169(16):1586-1596. doi:10.1016/j.jplph.2012.05.021.

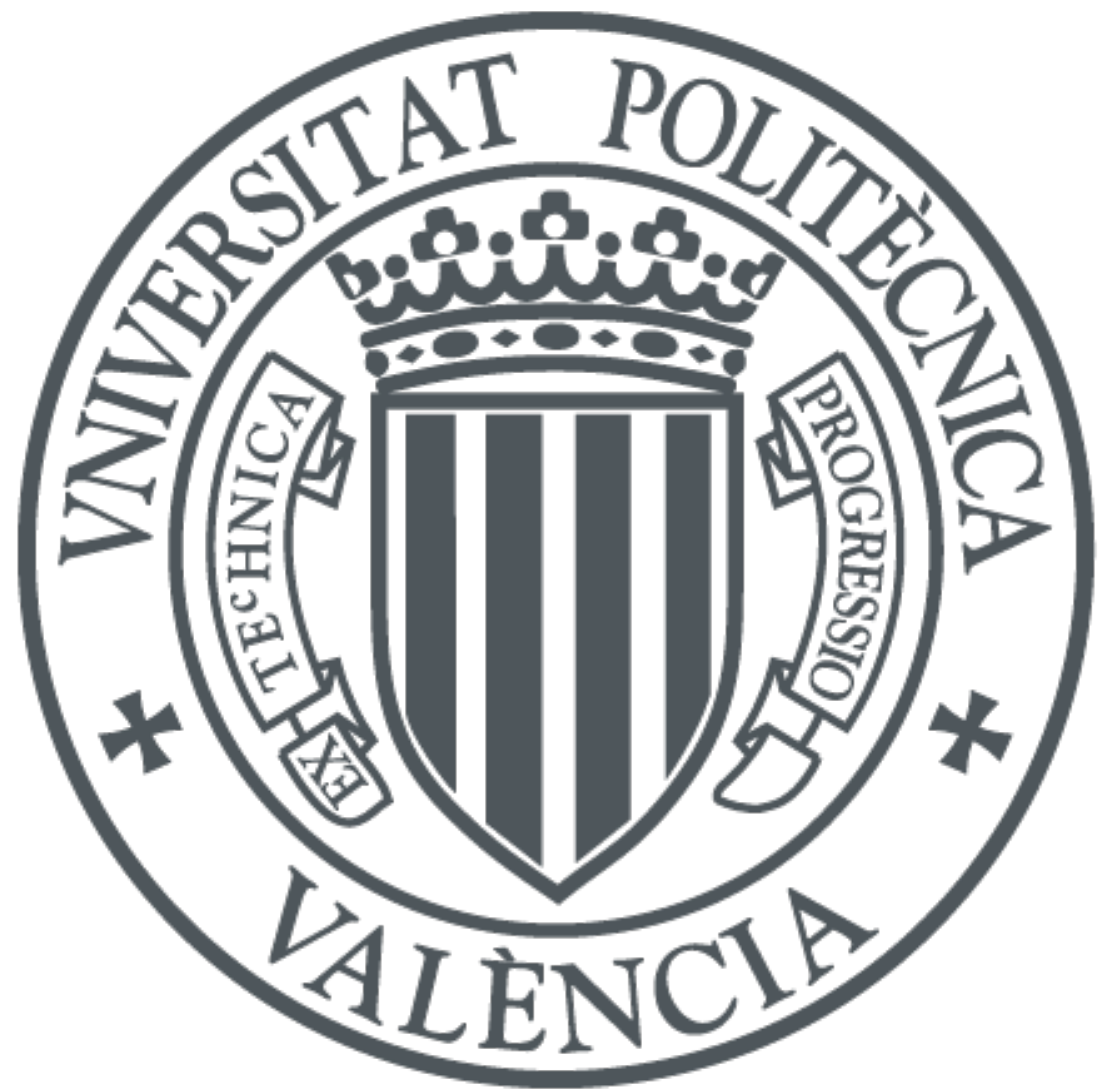

The final publication is available at

http://dx.doi.org/10.1016/j.jplph.2012.05.021

Copyright Elsevier

Additional Information 


\section{Metabolic fingerprinting of Tomato Mosaic Virus infected Solanum}

\section{lycopersicum}

5 M. Pilar López-Gresa a b, Purificación Lisón a , Hye Kyong Kim ${ }^{\text {b }}$, Young Hae Choi ${ }^{\text {b }}$, Robert Verpoorte ${ }^{\mathrm{b}}$, Ismael Rodrigo ${ }^{\mathrm{a}}$, José María Bellés ${ }^{\mathrm{a}}{ }^{*}$, and Vicente Conejero a

${ }^{a}$ Instituto de Biología Molecular y Celular de Plantas, Universidad Politécnica de Valencia-Consejo Superior de Investigaciones Científicas, Camino de Vera s/n 46022

Valencia, Spain.

${ }^{b}$ Natural Products Laboratory, Institute of Biology, Leiden University, Leiden, The Netherlands

15 * Correspondence to: José María Bellés, Instituto de Biología Molecular y Celular de Plantas, Universidad Politécnica de Valencia-Consejo Superior de Investigaciones Científicas, Camino de Vera s/n 46022 Valencia, Spain. E-mail: jmbelles@btc.upv.es 


\section{Abstract}

${ }^{1} \mathrm{H}$-nuclear magnetic resonance (NMR)-based metabolomics has been applied to study the compatible interaction between tomato plants and tomato mosaic virus (ToMV). A detailed time course of metabolic fingerprinting of ToMV-inoculated and noninoculated systemically infected tomato leaves has provided a fundamental understanding of the metabolic state of the plant not only in response to ToMV

25 infection, but also under various physiological conditions. By this analytical platform a total of 32 metabolites including amino/organic acids, sugars, phenylpropanoids, flavonoids and other miscellaneous compounds were detected. Using multivariate data analysis, we have identified a subset of metabolites induced during the plant defence response and metabolites whose accumulation was dependent on the developmental 30 stage, the position of the leaf on the stem, and the harvesting time. Specifically, a general time-dependent decrease in organic acids, amino acids (excluding asparagine), phenylpropanoids and rutin was observed in individual leaves. In addition, metabolite alterations were also found to correlate with the developmental stage of the leaf: high levels of organic acids, some amino acids, phenylpropanoids, and flavonoids were

35 found in lower leaves while elevated amounts of sugars were present in the upper ones. Moreover, a marked variation in the content of some metabolites was also observed to be associated to the asymptomatic ToMV infection both in inoculated and systemically infected leaves. While flavonoids accumulated in virus-inoculated leaves, increased levels of phenylpropanoids were observed in non-inoculated leaves where ToMV 40 actively replicates. Finally, diurnal changes in the metabolite content were also observed: an increase of amino acids and organic acids (except glutamic acid) were observed in the samples collected in the morning, whereas sugars and secondary metabolite levels increased in the tomato leaves harvested in the evening. 
45 Keywords: Solanum lycopersicum; Tomato Mosaic Virus (ToMV); plant-pathogen interaction; systemic acquired resistance (SAR); NMR-based metabolomics 


\section{Introduction}

Higher plants are, in most cases, passive receivers of biotic (viroids, viruses, bacteria,

50 fungi, nematodes or insects) and abiotic (salinity and drought) stresses. Consequently, they have evolved a large variety of sophisticated defence mechanisms to resist different types of stress (Dixon, 2001; Dangl and Jones, 2001).

Two types of plant-microbe interactions can be defined: incompatible and compatible. Incompatible interactions have been extensively studied principally because of their

55 direct and practical applications in the field (Vlot et al., 2009), whereas much less consideration has been paid to the compatible interactions (O'Donnell et al., 2001; Huang et al., 2003).

In an incompatible interaction, after an initial perception of the pathogen by the host, defences are rapidly activated resulting ultimately in the so-called hypersensitive response, which culminates in localized cell death at the area of pathogen inoculation and, concomitantly, the development of different degrees of necrosis (Ryals et al., 1996). The pathogen infection is thus limited to small parts immediately surrounding the initially infected cells and signals are generated which stimulate defensive reactions in uninfected areas of the plant. These distal sites then become more resistant to subsequent infections. This phenomenon, known as systemic acquired resistance (SAR), results in an immunization against future microbe attacks (Sticher et al., 1997; Durrant and Dong, 2004).

Frequently, a defensive response is also induced in a compatible interaction between a susceptible host and a virulent pathogen. However, in this case, in the absence of genefor-gene resistance, no necrosis occurs (Conejero et al., 1990; Dixon et al., 1994). In this type of interaction microbes actively multiply and spread throughout the host leading to disease and even the death of the infected plant. 
Although differing in the speed of the response to the pathogens, incompatible and compatible interactions share very similar general and common features, such as the induction of the plant signalling compounds, such as salicylic acid (SA; 2hydroxybenzoic acid) (Sticher et al., 1997; Vlot et al., 2009) and ethylene, and the synthesis of pathogenesis-defence proteins (van Kan et al., 1992; van Loon et al., 2006). One of the most well established defence responses in plants is the biosynthesis of an often complex array of natural chemicals belonging to phenolic metabolism (Hahlbrock and Scheel, 1989; Dixon et al., 2002; Abdel-Farid et al., 2009). A pending goal in molecular plant pathology is to characterize the metabolites that play a role in the defence response against pathogens. Traditionally, the analytical techniques employed in the search for these metabolites were basically chromatographic, coupled to spectroscopic detectors or mass spectrometers (Baumert et al., 2001; Shadle et al., 2003; Tan et al., 2004) . Accordingly, the range of metabolites that can be identified using these techniques is restricted based on the case specific experimental procedures employed and the physical-chemical properties of the compounds investigated (Roepenack-Lahaye et al., 2003; Bednarek et al., 2005; Fayos et al., 2006; Zacarés et al., 2007; Bellés et al., 2008; López-Gresa et al., 2011). However, a considerable amount of biomolecules with different biological functions (and physical-chemical properties), are proposed to be involved in the physiology of plant/pathogen interactions. Therefore, more robust analytical systems need to be developed in order to detect and accurately identify this considerable number of primary and secondary metabolites (Glauser et al., 2010). The introduction of so-called metabolomics has allowed the relatively rapid, and at the same time thorough, detection of a vast range of metabolites, thus providing an in-depth analysis of the total metabolome of biological processes in the plant. Although it is impossible for one single analytical method to completely characterize the entire plant 
metabolite profile, nuclear magnetic resonance spectroscopy (NMR) is an excellent tool to provide a macroscopic view of the majority of the components of the plant metabolome (Verpoorte et al., 2008; Broyart et al., 2010; Kim et al., 2010; Leiss et al., 2011). In fact, NMR-based metabolomics has been widely used to decode the broad range of chemical compounds that might be implicated in the plant defence against microbe attacks (Choi et al., 2006; Ward et al., 2010). Our group has been applying this technology to the detection and structure elucidation of metabolites in plants upon treatment with a wide range of biotic and abiotic stress agents (Choi et al., 2004; Simoh et al., 2009; Jahangir et al., 2009; Mirnezhad et al., 2010; Simoh et al., 2010). In recent years, very detailed NMR metabolomic studies on both compatible and incompatible plant-pathogen interactions have been reported (Lima et al., 2010; Bollina et al., 2010;

110 Ward et al., 2010; Jones et al., 2011).

Virus diseases cause serious losses worldwide in horticultural and agricultural crops (Lomonossoff, 1995). The cultivated tomato is subjected to a range of diseases resulting from infection with certain strains of tobacco mosaic virus. The mosaic disease in tomato is the most persistent virus in terms of its ability to survive outside plant cells

115 and in dead tissues (Broadbent, 1976). For this reason and because of its easy transmission this virus has a high rate of infectivity. ToMV is the most troublesome viral disease of tomatoes, causing distortion of leaves and fruit and stunting of growth. However, few metabolomic reports on plant/virus interactions have been described in the literature, and a complete analysis of metabolites has been carried out only in the 120 case of the hypersensitive response of tobacco plants to tobacco mosaic virus (TMV) (Choi et al., 2006). In our previous report, some characteristic metabolites of tomato plants related to either Citrus Exocortis viroid (CEVd) or Pseudomonas syringae infection have been identified by NMR-based metabolomics (López-Gresa et al., 2010). 
Tomato and Tobamovirus ToMV constitutes another model plant-virus interaction

125 system. But, as far as we know, no study on metabolic fingerprinting of tomato associated with plant development and its interaction with the compatible Tobamovirus ToMV has yet been published. Therefore, tomato plants infected by ToMV were studied at different times post-inoculation in order to extend our knowledge to additional plant/virus interactions.

130 Using the Solanum lycopersicum-ToMV interaction as a basic model system, we aim to reveal the role of defence metabolites involved in this infection by NMR spectroscopy combined with multivariate data analysis. As part of an ongoing global investigation on the metabolic state of tomato plants under different biotic or abiotic stress conditions, the main aim of this high throughout NMR-based metabolic analysis is geared to

135 increase the knowledge of the tomato ToMV infected leaf metabolome in diverse states of plant development and disease progression. A better understanding of the tomato defence response might also provide relevant data for metabolic engineering of tomato plants with higher resistance towards pathogens.

\section{Experimental}

Plant material and inoculation procedure

Seeds from tomato (Solanum lycopersicum cv. Rutgers) (Western Hybrid Seeds Inc., 145 Hamilton City, CA, U.S.A) previously surface-sterilized with bleach were used in this work. Extreme care was taken in order to maintain controlled conditions of growth to be sure that the variables of study were limited to the effect of the infection and the natural growth of the plants. Experimental lots of 120 plants of similar morphological and physiological characteristics were prepared for the experiment. The plants (one per pot) 
were grown in 15-cm-diameter pots containing a mixture of peat (Biolan) and vermiculite 1:1 and were subirrigated once a day. The tomato plants were maintained in a controlled growth chamber at a constant temperature of $24{ }^{\circ} \mathrm{C}(12 \mathrm{~h}$ photoperiod) and with relative humidity ranging from $60 \%$ (day) to $70 \%$ (night). Five-week-old Rutgers tomato plants at the five- to six-leaf stage were used in all the experiments described in

155 this article. Infection of Rutgers tomato plants with the Murakishi PV-0143 (DSMZ) strain of Tomato Mosaic Virus was performed with a viral extract obtained from leaves of ToMV-infected tomato plants that were homogenized in $10 \mathrm{mM}$ phosphate buffer (pH 7.2), 0.5\% sodium bisulfite, $0.5 \%$ diethyldithiocarbamic acid (1 g of leaf material in $20 \mathrm{~mL}$ of buffer) as described (Bellés et al., 2006). The third leave (leaf position numbered from the base to the apex) of tomato plants were dusted with carborundum (particle size $0.037 \mathrm{~mm}$ ). One $\mathrm{ml}$ of viral extract or buffer (mock-inoculated control) was applied by gently rubbing the upper face of the third leaf. The third and fourth leaves were harvested separately at day $1,2,3,4,5,7,9,11,13$, and 16 postinoculation at $9 \mathrm{am}(9: 00 \mathrm{~h})$ and 7 pm (19:00 h) each day. Samples were immediately

165 frozen in liquid nitrogen, subsequently ground in a cooled mortar and lyophilized for metabolomic studies. Three biological replicates from both mock- and ToMVinoculated tomato plants were analyzed for each time point.

\section{Protein extraction and electrophoretic analysis}

Leaves from mock-inoculated and ToMV-infected tomato leaves were harvested at the indicated times and rapidly frozen in liquid nitrogen and stored at $-80^{\circ} \mathrm{C}$ until use. Protein extracts of tomato leaves were performed by homogenization in extraction buffer (50mM Tris, pH 7.5, 15mM mercaptoethanol), as described in (Rodrigo et al., 
175 1993). Proteins were separated by SDS-PAGE and stained with Coomassie Brilliant Blue R-250, as described (Conejero and Semancik, 1977).

\section{Extraction and NMR spectra measurements}

180 Twenty five mg of freeze-dried plant material were extracted in $2 \mathrm{~mL}$-Eppendorf tubes with $1 \mathrm{~mL}$ of a mixture of $\mathrm{KH}_{2} \mathrm{PO}_{4}$ buffer (pH 6) in $\mathrm{D}_{2} \mathrm{O}$ containing $0.05 \%$ trimethylsilane propionic acid sodium salt (TMSP) and $\mathrm{CH}_{3} \mathrm{OH}-d_{4}(1: 1)$. The extracts were vortexed, vigorously, sonicated for $20 \mathrm{~min}$ and then centrifuged at 13,000 rpm for $10 \mathrm{~min}$. Seven hundred $\mu \mathrm{L}$ of the supernatant were transferred in $5 \mathrm{~mm}-\mathrm{NMR}$ tubes for 185 the spectral analysis.

${ }^{1} \mathrm{H}$ NMR spectra were recorder at $25{ }^{\circ} \mathrm{C}$ on a $600 \mathrm{MHz}$ Bruker AV 600 spectrometer equipped with cryo-probe operating at a proton NMR frequency of $600 \mathrm{MHz}$. Methyl signal of $\mathrm{CH}_{3} \mathrm{OH}-d_{4}$ was used as the internal lock. Each ${ }^{1} \mathrm{H}$ NMR spectrum consisted of 128 scans requiring $10 \mathrm{~min}$ acquisition time with the following parameters: 0.25 $\mathrm{Hz} /$ point, pulse width $(\mathrm{PW})=30^{\circ}(10.8 \mu \mathrm{s})$, and relaxation delay $(\mathrm{RD})=1.5 \mathrm{~s}$. A presaturation sequence was used to suppress the residual $\mathrm{H}_{2} \mathrm{O}$ signal with low power selective irradiation at the $\mathrm{H}_{2} \mathrm{O}$ frequency during the recycle delay. Free induction decay (FIDs) were Fourier transformed with Line Broadening (LB) $=0.3 \mathrm{~Hz}$ and the spectra were zero-filled to $32 \mathrm{~K}$ points. The resulting spectra were manually phased and

195 baseline corrected, and calibrated to TMSP at $0.0 \mathrm{ppm}$, using Topspin (version 2.1, Bruker).

\section{Data analysis}


${ }^{1} \mathrm{H}$ NMR spectra were automatically reduced to ASCII files using AMIX (v. 3.7, Bruker Biospin). Spectral intensities were scaled to total intensity TMSP and reduced to integrated regions of equal width $(0.04 \mathrm{ppm})$ corresponding to the region of $\delta 0.4-\delta$ 10.00. The region of $\delta 4.7-\delta 4.9$ was excluded from the analysis because of the residual signal of water as well as $\delta 3.28$ - $\delta 3.34$ for residual methanol. Partial least square (PLS) was performed with the SIMCA-P software (v. 11.0, Umetrics, Umeå, Sweden) using unit variance (UV) scaling method.

\section{Results and Discussion}

The ToMV strain employed in this work failed to induce visible symptoms in the tolerant tomato cultivar Rutgers during the 16 day experiment. Only subtle curling, slight distortions and malformations of the leaves and a very limited stunting of the

215 plants were observed at long periods post-inoculation. To monitor the progress of the ToMV disease in the infected tomato plants, a polyacrylamide gel electrophoresis (SDS-PAGE) of protein extracts from the third-inoculated and systemically fourthinfected leaves was performed to detect the viral capsid accumulation. The knowledge of the virus accumulation pattern at each time point allows us to relate the metabolic changes observed during the infection with the amount of virus present. ToMV actively multiplied in the inoculated leaf, and the 17-kDa ToMV coat protein could be clearly detected at day three post-inoculation, as assessed by electrophoretic analysis (Fig. 1). In the inoculated leaves, ToMV accumulation increased in a time-dependent manner, reaching its maximum level at 5 day post-inoculation, and decreasing thereafter (data not shown). The virus started to spread up to the fourth leaf by day 7 , following a 
similar induction kinetic pattern to the inoculated leaves and remaining constant from day 11 until the end of the 16-day experiment (Fig. 1). The phenotypic alterations observed consisted mainly of gradual development of brown necrotic spots at the inoculation site (produced by rubbing the leaves with carborundum in the inoculation zone) in both the inoculated and mock-inoculated leaves.

\section{Alterations of the metabolic profiles of leaves induced by ToMV infection and developmental conditions}

235 Diverse factors such as light intensity, temperature, water, nutritional status can produce notable effects on the metabolome during the life cycle of the plant. In metabolic studies it is crucial to be able to differentiate the changes that result from uncontrolled factors and those that are genuinely caused by the experimental manipulation being studied. In the present work, we studied the leaf metabolome affected by ToMV infection. The experimental conditions were carefully designed and controlled to ensure that the metabolic differences identified were restricted to only the effect of ToMV infection. However, in addition, we expanded our study to include other non-controllable factors such as plant aging and development stage. Interestingly, these factors had very important effects on the metabolome of tomato leaves.

245 Plant metabolites have very diverse physical-chemical properties and are present in a wide range of quantities (Wolfender, 2009). Therefore, the solvent extraction used for their isolation from the plant tissues should be able to rapidly and efficiently dissolve metabolites in an unbiased manner. In this work, tomato leaf metabolites were extracted using a single-step extraction method consisting of a polar solvent mixture of methanol and a buffered aqueous solution. This method is practical, is not time consuming and has been shown to possess a high extraction efficiency constitutes an optimized 
extraction protocol for polar untargeted metabolites (Hendrawati et al., 2006; Verpoorte et al., 2007; Verpoorte et al., 2008). NMR spectroscopy in combination with multivariable data analysis was performed on control and ToMV-infected tomato leaves in order to determine the metabolic changes during this plant-pathogen interaction. The first step of NMR metabolomic studies is the analysis of the plant extract spectra (Choi et al., 2006). ${ }^{1} \mathrm{H}$ NMR spectra, 2D NMR experiments (2D J-resolved, COSY and HMBC), our in-house database of reference compounds and previous reports (LópezGresa et al., 2010) were used for the signal assignment of metabolites from the tomato leaves. Table 1 shows a list of 32 the identified metabolites including 13 aminoacids, 5 organic acids (3 citrate-cycle intermediates), 3 sugars, 4 phenylpropanoids, 1 flavonoid, and 6 miscellaneous compounds with their characteristic chemical shifts and coupling constants.

After the chemical analysis of the spectra, the following step in a metabolomic study is the comparison of all the samples through multivariate data analysis (MVDA). The first step of the statistical analysis of these large data sets (in this case 240 samples), was the application of a principal component analysis (PCA) in order to group the plants according to the NMR signals characteristic for the metabolic changes caused by the ToMV infection. In the PCA score plot, healthy and infected samples were grouped together, showing that only slight metabolic changes were produced by the infection. Other parameters, such as time (from 1 to 16 days post inoculation (dpi), hereafter considered as age), position in the stem (lower and upper leaves) or collection time (9:00 h and 19:00 h) play an important role in determining the metabolome, even more than the infection. According to this observation, a partial least square analysis (PLS) was carried out defining as the $\mathrm{X}$ variable the area of binned ${ }^{1} \mathrm{H}$-NMR signals and as stepwise Y variables age, developmental stage, infection, and sample collection 
time. A clear separation was obtained between: a) age of the leaves by component 1 (Fig 2): b) leaves located at different positions in the stem by component 2 (Fig 2); c) healthy control and ToMV infected leaves by component 3 (Fig 3); and d) samples collected at 9:00 h and 19:00 h by component 4 (Fig 4). In order to differentiate the identified compounds, a loading plot was used in which the correlation between grouping and correlated metabolites was shown (Table 1).

285

The first component of PLS explained the changes in the chemical composition during the time course of the experiment (age of the plant: 1 to $16 \mathrm{dpi}$ ). Analysis of loading column plot of PLS component 1 showed a time-dependent increase in the content of sugars and a decline of the organic and amino acids, except for asparagine. This observation is in agreement with the results obtained examining the amount of several amino acids in different lines of Arabidopsis during the leaf senescence (Diaz et al., 2005). Only asparagine was higher in “old” leaves (from 9 to 16 dpi), in accordance with the well-known decrease of aspariginase activity observed during leaf maturation (Sieciechowicz et al., 1988) (Fig 2 and Table1). The increase of sugars observed in the “old” leaves may be correlated to the increase of total surface area in all leaves with age, which augments the rate of photosynthesis, thus producing a greater accumulation of carbohydrates (Abdel-Farid et al., 2009). Important concentrations of phenylpropanoids and the flavonoid, rutin were found in “young” leaves (from 1 to 9 dpi). These secondary metabolites are associated with defence mechanisms and their 300 presence in the "young” leaves reflects the need of this tissues to increase their defensive potential (Brown et al., 2003; Baker et al., 2010). According to the optimal plant defence theory, plants tend to allocate more defence-associated metabolites to the 
valuable plant parts during development, to protect them from stress factors and to maximize their fitness (Kaur et al., 2010).

Examination of the component 2-PLS loading plot showed the metabolites which strongly contributed to the separation of the metabolic profiles based on the position of the leaf on the stem. Lower (third) leaves were placed on the positive side of PLS component 2, whereas immediately upper (fourth) leaves were on the negative side (Fig 2). When comparing the metabolome of the leaves located at different positions in the stem, it was observed that organic acids (malic, citric, succinic, fumaric, and formic acid), amino acids (GABA, glutamic, glutamine, aspartic, asparagine, tryptophan, and phenylalanine), phenylpropanoids, and flavonoids were induced in lower leaves, while upper leaves showed higher amount of sugars, acetic acid, choline, trigonelline and the aliphatic amino acids arginine, alanine, and valine (Table 1). Many primary metabolites were induced as a consequence of the abrasive carborundum applied during inoculation process, together with secondary metabolites, in local leaves (Dixon and Paiva, 1995). However, sucrose and glucose levels decreased in these lower leaves. Presumably, carbohydrate consumption is required for production of energy to support the biosynthesis of defensive phenolic compounds induced by wounding (Broeckling et al., 2005). Since the metabolite content varies throughout the plant (Choi et al., 2004), it is crucial to harvest the leaves in the same leaf position in order to accurately compare metabolite fingerprinting of infected with the corresponding equivalent control leaves. Focused on our aim, which is to understand the tomato defence reaction against ToMV infection, a detailed metabolic study was performed examining component 3 of the

325 PLS. The score plot for component 1 versus component 3 of PLS from the ${ }^{1} \mathrm{H}$ NMR spectra shows that ToMV-infected leaves are separated from healthy ones, independently of whether they were directly inoculated with the virus or systemically infected (Fig 3). The separation is mainly due to component 3, however, both aging and 
stem position produce a shift along component 1 . To exclude differences due to this biological variability, partial least square-discriminant analysis (PLS-DA) was applied separately on 1) mock-inoculated (third) versus the ToMV locally-infected (third) leaves until eleven dpi (Fig 5A) and 2) upper-mock-inoculated (fourth) versus systemically-infected (fourth) leaves from five to thirteen dpi (Fig 5B). Applying this statistical analysis, a good separation was observed between local and systemically 335 infected leaves.

Local infection was characterized by component 1 (Fig 5A), in which rutin, glucose, choline, GABA, glutamine, asparagine, phenylalanine, glutamic, aspartic, malic, citric, formic, and fumaric acid, were found to be the major contributing metabolites (Table 1). Determination of the relative abundance of several amino acids in ToMV-infected tomato leaves revealed a decrease in the content of some of them and an increase in others. The results previously found in $P$ syringae-infected-tomato leaves, in which in general high levels of some amino acids were found, (Pérez Garcia et al., 1998; LópezGresa et al., 2010), are in contrast with those found in this work upon viral infection which produced a general decrease in the pool of amino acids. Only

345 glutamine/glutamate and asparagine/aspartate were clearly induced in leaves inoculated with ToMV. It is well established that glutamine may act as substrate for asparagine biosynthesis, so their levels could be interconnected (Eason et al., 1996). These results are in accordance with previous reports suggesting that glutamate is a precursor of stress-related compounds in plants (Hothorn et al., 2006) and that glutamine content was higher in Vitis vinifera plants resistant to mildew (Figueiredo et al., 2008). In this context, it is pertinent to point out that opposite results have been reported in some plant pathogen interactions. Thus, as observed in the present work (Table 1), and previously (Pérez-García et al., 1998; López-Gresa et al., 2010), the alanine content significantly decreases in tomato leaves after systemic infection with viral or bacterial pathogens. By 
contrast, alanine exhibited the largest increase by far in rice plants upon infection with the fungal pathogen Magnaporthe gerisea (Jones et al. 2010), and as such, alanine is considered as a key marker in this compatible interaction.

GABA induction in plants in response to an abiotic stress treatment is a widely observed phenomenon (Wallace et al., 1984; Mayer et al., 1990; Allan et al., 2008). Our results agree with a previously published study of the metabolic changes in vitis plants affected by the Esca disease. In this report, the authors identified a marked increase in GABA as a clear indicator of healthy or diseased leaves (Lima et al., 2010). Moreover, increases in phenylalanine, which is the substrate of the key enzyme of the phenylpropanoid biosynthesis pathway, phenylalanine ammonia lyase (PAL), correlated 365 well with the concomitant induction of phenylpropanoid pathway products. The positive relationship between sucrose and UDP-glucose may be explained because both metabolites can be reversibly formed by the sucrose synthase enzyme. The primary metabolites, malic and citric acid were found in the present study to be altered in both locally inoculated and systemically -infected tomato leaves. Our results are consistent with those of Choi et al. (2006), studying the hypersensitive interaction between tobacco and tobacco mosaic virus (TMV). The authors reported that these organic acids also accumulated in both TMV-inoculated and in SAR tobacco leaves.

The study of phenolic compounds during the progression of infection is very relevant based on their proposed defensive role in plants. The flavonoid, rutin, which has been 375 related to the enhancement of the defence system against some stress conditions (Suzuki et al., 2005), clearly increased in ToMV-infected tomato leaves. Previously, we had also found that citrus exocortis viroid and $P$. syringae also elicited the synthesis of this compound in tomato leaves (López-Gresa et al., 2010). Unexpectedly, caffeoyl esters of polyhydroxy compounds (glucaric acid) did not accumulate in locally infected tomato leaves, even though phenylpropanoid metabolism is activated under various stress 
conditions, such as UV irradiation, mechanical wounding, or pathogen attack (Lawton and Lamb, 1987; Dixon and Lamb, 1990). In fact, TMV-infected tobacco plants showed, among the phenylpropanoids, only 5-O-caffeoylquinic acid accumulation in the locally infected leaves (Choi et al., 2006). Additionally, a decrease content of tryptophan was observable in these ToMV-inoculated leaves. Products of the tryptophan pathway are metabolized into auxin, glucosinolates, phytoalexins, alkaloids, and other indolic compounds, which play diverse roles in plant biological processes, including plant-pathogen interaction (Radwanski and Last, 1995). Previously, NMR spectroscopybased metabolomics had shown that the levels of a natural derivative of salicylic acid, 2,5-dihydroxybenzoic acid (gentisic acid), markedly increased as a conjugated form named gentisic acid 5-O- $\beta$-D-xyloside in tomato leaves infected by the citrus exocortis viroid. Infection with this viroid causes strong symptoms (extreme epinasty, curling, and rugosity of leaf and leaflets) and the intensity of symptoms exhibited a good correlation with gentisic acid content in viroid- and virus-infected plants (Bellés et al., 1999; Bellés et al., 2006). It is therefore not surprising that the present asymptomatic infection caused by ToMV did not induce a significant accumulation of gentisic acid. Viruses produce a vast array of symptoms in plants, and many of them appear to be common even among combinations between diverse virus and plants. Some virusinfected plants may show no symptoms, as in the case of Rutgers tomato infected by this strain of ToMV, and consequently, are excellent virus carriers, as they go unnoticed in the greenhouse. Symptoms could also be influenced by several factors such as environmental conditions, plant age at infection or virulence of the virus. The present NMR based metabolomic study not only gives important and broad physiological information, but also points out that care must be taken to select virus-free plants when performing metabolic analysis. 
Metabolic alterations of non-inoculated systemic leaves were compared with the corresponding controls analogously to locally infected leaves by PLS-DA. In this case, upper healthy and ToMV systemically infected leaves from five to eleven thirteen dpi were used as discrete classes (Fig 5B). The selected time course was based on the time required to detect viral capsid accumulation in distal leaves (Fig1). In this case, for the identification of the metabolites involved in systemic defence response after virus inoculation, the loading plot of component 1 was analyzed. High concentrations of phenylpropanoids, rutin, choline, organic acids, sucrose, GABA, tryptophan, phenylalanine, glutamic, and aspartic acid were characteristic of systemically infected

415 leaves. Only glutamine and asparagine were not induced in these leaves compared with inoculated ones. Unexpectedly, in the upper control leaves there was an increase in the levels of some metabolites with signals at $\delta 8.02,7.90,7.42,6.78,6.14$, and 6.10, belonging to some flavonoid glycosides, which probably have a 4'-OH in the $\mathrm{B}$ ring (Fig 6).

420 All these results suggest that flavonoids might be involved in the local response, while phenylpropanoids could play a defensive role in the distal leaves. In a previous study, Niehl et al. (2006) reported a detailed analysis of defence responses during the compatible interaction of potato plants between potato (Solanum tuberosum L. cv Desiree) and the Potato Virus X (PVX). As in Rutgers tomato plants infected with 425 Tomato Mosaic Virus (ToMV), infection of potato with PVX results in a systemic infection of the plants. In agreement with the results presented here, the authors found that low molecular weight secondary metabolites such as $\beta$-phenylethylamine-alkaloids were substantially induced upon PVX infection. By contrast, primary metabolism as levels of organic acids (citrate-cycle intermediates), sugars, and aminoacids remained mostly unchanged in PVX-infected potato leaves, this being in contrast with the results observed in ToMV-infected Rutgers tomato leaves (Table 1). Taken together, all these 
results demonstrate that potato and tomato respond to systemic virus infection in a different way depending of the host plant.

Finally, the metabolic alterations due to sample collection time were identified by observed between tomato leaves collected at 9:00 $\mathrm{h}$ and 19:00 $\mathrm{h}$, mainly from the youngest plants (from 1 to 9 days post-inoculation). It is well known that fluctuations of primary and secondary metabolites are observed when plants are harvested at different times of the day. NMR metabolomic analysis of Cannavis sativa clearly demonstrates a clear difference between samples harvested in the morning and afternoon (Kim and Verpoorte, 2010). In our study, amino acids and organic acids (except glutamic acid) were induced in the samples collected in the morning, while sugars and secondary metabolites were induced in the tomato leaves harvested in the evening. This carbohydrate production is likely a result of the photosynthesis that takes place during

445 the day. A complete quantitative analysis of carbohydrates and primary metabolites was done through a diurnal period in potato leaves (Urbanczyk-Wochniak et al., 2005). Although it is difficult to compare our results with those obtained in potato because we have not performed a quantitative analysis, levels of carbohydrates, particularly sucrose, and citric acid followed the same trend as in potato leaves.

450 As shown in this work, a broad variety of primary and secondary compounds can be detected by using simple extraction procedures and NMR spectroscopy. Multivariate data analysis methods, such as partial least square (PLS) and partial least squarediscriminant analysis (PLS-DA) were applied to characterize the metabolic alterations of infected symptomless tomato leaves, taking into account their development stage and 455 the time of harvesting.

\section{Acknowledgements}


460 The authors are grateful to Cristina Torres Vidal for technical support and Dr. Lynne Yenush for critical reading of the manuscript and exciting intellectual discussions. This work was supported by Grant BFU 2009-11958 from Secretaría de Estado de Investigación from Spanish Ministry of Science and Innovation. M.P.L.G held a postdoctoral fellowship JAEDoc 0800402 from the Consejo Superior de 465 Investigaciones Científicas (Spain) and was recipient of a postdoctoral fellowship (BEST/2010/111) from Generalitat Valencia (Spain). 


\section{References}

Abdel-Farid IB, Jahangir M, van den Hondel CAMJ, Kim HK, Choi YH, Verpoorte R. Fungal infection-induced metabolites in Brassica rapa. Plant Science 2009; 176: 608615.

Allan WL, Simpson JP, Clark SM, Shelp BJ. Gamma-hydroxybutyrate accumulation in Arabidopsis and tobacco plants is a general response to abiotic stress: putative regulation by redox balance and glyoxylate reductase isoforms. Journal of Experimental Botany 2008; 59: 2555-2564.

Baker C, Owens RA, Whitaker BD, Mock NM, Roberts DP, Deahl KL, Aver'yanov AA. Effect of viroid infection on the dynamics of phenolic metabolites in the apoplast of tomato leaves. Physiological and Molecular Plant Pathology 2010; 74: 214-220.

Baumert A, Mock HP, Schmidt J, Herbers K, Sonnewald U, Strack D. Patterns of phenylpropanoids in non-inoculated and potato virus Y-inoculated leaves of transgenic tobacco plants expressing yeast-derived invertase. Phytochemistry 2001; 56: 535-541.

Bednarek P, Schneider B, Svatos A, Oldham NJ, Hahlbrock K. Structural complexity, differential response to infection, and tissue specificity of indolic and phenylpropanoid secondary metabolism in Arabidopsis roots. Plant Physiology 2005; 138: 1058-1070.

Bellés JM, Garro R, Fayos J, Navarro P, Primo J, Conejero V. Gentisic acid as a pathogen-inducible signal, additional to salicylic acid for activation of plant defenses in tomato. Molecular Plant-Microbe Interactions 1999; 12: 227-235.

490 Bellés JM, Garro R, Pallas V, Fayos J, Rodrigo I, Conejero V. Accumulation of gentisic acid as associated with systemic infections but not with the hypersensitive response in plant-pathogen interactions. Planta 2006; 223: 500-511.

Bellés JM, López-Gresa MP, Fayos J, Pallas V, Rodrigo I, Conejero V. Induction of cinnamate 4-hydroxylase and phenylpropanoids in virus-infected cucumber and melon plants. Plant Science 2008; 174: 524-533.

Bollina V, Kumaraswamy G, Kushalappa AC, Choo TM, Dion Y, Rioux S, Faubert D, Hamzehzarghani H. Mass spectrometry-based metabolomics application to identify quantitative resistance-related metabolites in barley against Fusarium head blight. Molecular Plant Pathology 2010; 11: 769-782.

500 Broadbent L. Epidemiology and Control of Tomato Mosaic-Virus. Annual Review of Phytopathology 1976; 14: 75-96.

Broeckling CD, Huhman DV, Farag MA, Smith JT, May GD, Mendes P, Dixon RA, Sumner LW. Metabolic profiling of Medicago truncatula cell cultures reveals the effects of biotic and abiotic elicitors on metabolism. Journal of Experimental Botany 2005; 56: 323-336.

Brown PD, Tokuhisa JG, Reichelt M, Gershenzon J. Variation of glucosinolate accumulation among different organs and developmental stages of Arabidopsis thaliana. Phytochemistry 2003; 62: 471-481. 
Broyart C, Fontaine JX, Molinie R, Cailleu D, Terce-Laforgue T, Dubois F, Hirel B, Mesnard F. Metabolic profiling of maize mutants deficient for two glutamine synthetase isoenzymes using ${ }^{1} \mathrm{H}-\mathrm{NMR}$-based metabolomics. Phytochem Anal 2010; 21: 102-109.

Choi HK, Choi YH, Verberne M, Lefeber AWM, Erkelens C, Verpoorte R. Metabolic fingerprinting of wild type and transgenic tobacco plants by ${ }^{1} \mathrm{H}-\mathrm{NMR}$ and multivariate analysis technique. Phytochemistry 2004; 65: 857-864.

515 Choi YH, Kim HK, Linthorst HJM, Hollander JG, Lefeber AWM, Erkelens C, Nuzillard JM, Verpoorte R. NMR metabolomics to revisit the tobacco mosaic virus infection in Nicotiana tabacum leaves. Journal of Natural Products 2006; 69: 742-748.

Conejero V, Bellés JM, García-Breijo F, Garro R, Hernández-Yago J, Rodrigo I, Vera P. Signalling in viroid pathogenesis. In: RSS Fraser, editor. Recognition and response in plant-virus interactions Springer-Verlag, Heidelberg, 1990, pp. 233-261.

Conejero V, Semancik JS. Exocortis Viroid - Alteration in Proteins of GynuraAurantiaca Accompanying Viroid Infection. Virology 1977; 77: 221-232.

Dangl JL, Jones JDG. Plant pathogens and integrated defence responses to infection. Nature 2001; 411: 826-833.

525 Diaz C, Purdy S, Christ A, Morot-Gaudry JF, Wingler A, Masclaux-Daubresse CL. Characterization of markers to determine the extent and variability of leaf senescence in Arabidopsis. A metabolic profiling approach. Plant Physiology 2005; 138: 898-908.

Dixon RA. Natural products and plant disease resistance. Nature 2001; 411: 843-847.

Dixon RA, Achnine L, Kota P, Liu CJ, Reddy MSS, Wang LJ. The phenylpropanoid 530 pathway and plant defence - a genomics perspective. Mol Plant Pathol 2002; 3: 371390 .

Dixon RA, Harrison MJ, Lamb CJ. Early events in the activation of plant defense responses. Annu Rev Phytopathol 1994; 32: 479-501.

Dixon RA, Lamb CJ. Molecular communication in interactions between plants and 535 microbial pathogens. Annual Review of Plant Physiology and Plant Molecular Biology 1990; 41: 339-367.

Dixon RA, Paiva NL. Stress-induced phenylpropanoid metabolism. Plant Cell 1995; 7: 1085-1097.

Durrant WE, Dong X. Systemic acquired resistance. Annual Review of Phytopathology 2004; 42: 185-209.

Eason JR, ODonoghue EM, King GA. Asparagine synthesis and localization of transcripts for asparagine synthetase in tips of harvested asparagus spears. Journal of Plant Physiology 1996; 149: 251-256.

Fayos J, Bellés JM, López-Gresa MP, Primo J, Conejero V. Induction of gentisic acid 5$545 O$-beta- $D$-xylopyranoside in tomato and cucumber plants infected by different pathogens. Phytochemistry 2006; 67: 142-148. 
Figueiredo A, Fortes AM, Ferreira S, Sebastiana M, Choi YH, Sousa L, Acioli-Santos B, Pessoa F, Verpoorte R, Pais MS. Transcriptional and metabolic profiling of grape (Vitis vinifera L.) leaves unravel possible innate resistance against pathogenic fungi. Journal of Experimental Botany 2008; 59: 3371-3381.

Glauser G, Boccard J, Rudaz S, Wolfender JL. Mass spectrometry-based metabolomics oriented by correlation analysis for wound-induced molecule discovery: identification of a novel jasmonate glucoside. Phytochem Anal 2010; 21: 95-101.

Hahlbrock K, Scheel D. Physiology and molecular-biology of phenylpropanoid metabolism. Annual Review of Plant Physiology and Plant Molecular Biology 1989; 40: 347-369.

Hendrawati O, Yao QQ, Kim HK, Linthorst HJM, Erkelens C, Lefeber AWM, Choi YH, Verpoorte R. Metabolic differentiation of Arabidopsis treated with methyl jasmonate using nuclear magnetic resonance spectroscopy. Plant Science 2006; 170: 1118-1124.

Hothorn M, Wachter A, Gromes R, Stuwe T, Rausch T, Scheffzek K. Structural basis for the redox control of plant glutamate cysteine ligase. Journal of Biological Chemistry 2006; 281: 27557-27565.

Huang J, Cardoza YJ, Schmelz EA, Raina R, Engelberth J, Tumlinson JH. Differential 565 volatile emissions and salicylic acid levels from tobacco plants in response to different strains of Pseudomonas syringae. Planta 2003; 217: 767-775.

Jahangir M, Abdel-Farid IB, Kim HK, Choi YH, Verpoorte R. Healthy and unhealthy plants: The effect of stress on the metabolism of Brassicaceae. Environmental and Experimental Botany 2009; 67: 23-33.

570 Jones OA, Maguire ML, Griffin JL, Jung YH, Shibato J, Rakwal R, Agrawal GK, Jwa NS. Using metabolic profiling to assess plant-pathogen interactions: an example using rice (Oryza sativa) and the blast pathogen Magnaporthe grisea. European Journal of Plant Pathology 2011; 129: 539-554.

Kaur H, Heinzel N, Schottner M, Baldwin IT, Galis I. R2R3-NaMYB8 regulates the accumulation of phenylpropanoid-polyamine conjugates, which are essential for local and systemic defense against insect herbivores in Nicotiana attenuata. Plant Physiology 2010; 152: 1731-1747.

Kim HK, Choi YH, Verpoorte R. NMR-based metabolomic analysis of plants. Nature Protocols 2010; 5: 536-549.

580 Kim HK, Verpoorte R. Sample preparation for plant metabolomics. Phytochem Anal 2010; 21: 4-13.

Lawton MA, Lamb CJ. Transcriptional activation of plant defense genes by fungal elicitor, wounding, and infection. Molecular and Cellular Biology 1987; 7: 335-341.

Leiss K, Choi Y, Verpoorte R, Klinkhamer P. An overview of NMR-based metabolomics to identify secondary plant compounds involved in host plant resistance. Phytochemistry Reviews 2011; 10: 205-216. 
Lima MR, Felgueiras ML, Graca G, Rodrigues JE, Barros A, Gil AM, Dias AC. NMR metabolomics of esca disease-affected Vitis vinifera cv. Alvarinho leaves. Journal of Experimental Botany 2010; 61: 4033-4042.

590 Lomonossoff GP. Pathogen-Derived Resistance to Plant-Viruses. Annual Review of Phytopathology 1995; 33: 323-343.

López-Gresa MP, Maltese F, Bellés JM, Conejero V, Kim HK, Choi YH, Verpoorte R. Metabolic response of tomato leaves upon different plant-pathogen interactions.

Phytochem Anal 2010; 21: 89-94.

595 López-Gresa MP, Torres C, Campos L, Lison P, Rodrigo I, Bellés JM, Conejero V. Identification of defence metabolites in tomato plants infected by the bacterial pathogen Pseudomonas syringae. Environmental and Experimental Botany 2011; 74: 216-228.

Mayer RR, Cherry JH, Rhodes D. Effects of heat-shock on amino-acid-metabolism of cowpea cells. Plant Physiology 1990; 94: 796-810.

600 Mirnezhad M, Romero-Gonzalez RR, Leiss KA, Choi YH, Verpoorte R, Klinkhamer PG. Metabolomic analysis of host plant resistance to thrips in wild and cultivated tomatoes. Phytochem Anal 2010; 21: 110-117.

Niehl A, Lacomme C, Erban A, Kopka J, Krämer U, Fisahn J. Systemic Potato virus X infection induces defence gene expression and accumalation of $\beta$-phenylethylamine605 alkaloids in potato. Functional Plant Biology 2006; 33: 593-604.

O'Donnell PJ, Jones JB, Antoine FR, Ciardi J, Klee HJ. Ethylene-dependent salicylic acid regulates an expanded cell death response to a plant pathogen. Plant J 2001; 25: 315-323.

Pérez-García A, Pereira S, Pissarra J, Gutiérrez AG, Cazorla FM, Salema R, de Vicente 610 A, Cánovas FM. Cytosolic localization in tomato mesophyll cells of a novel glutamine synthetase induced in response to bacterial infection or phosphinothricin treatment. Planta 1998; 206: 426-434.

Radwanski ER, Last RL. Tryptophan Biosynthesis and Metabolism - Biochemical and Molecular-Genetics. Plant Cell 1995; 7: 921-934.

615 Rodrigo I, Vera P, Tornero P, Hernandezyago J, Conejero V. cDna cloning of viroidinduced tomato pathogenesis-related protein-p23 - characterization as a vacuolar antifungal factor. Plant Physiology 1993; 102: 939-945.

Roepenack-Lahaye E, Newman MA, Schornack S, Hammond-Kosack KE, Lahaye T, Jones JDG, Daniels MJ, Dow JM. p-coumaroylnoradrenaline, a novel plant metabolite implicated in tomato defense against pathogens. Journal of Biological Chemistry 2003; 278: 43373-43383.

Ryals JA, Neuenschwander UH, Willits MG, Molina A, Steiner HY, Hunt MD. Systemic acquired resistance. Plant Cell 1996; 8: 1809-1819.

Shadle GL, Wesley SV, Korth KL, Chen F, Lamb C, Dixon RA. Phenylpropanoid 625 compounds and disease resistance in transgenic tobacco with altered expression of Lphenylalanine ammonia-lyase. Phytochemistry 2003; 64: 153-161. 
Sieciechowicz KA, Joy KW, Ireland RJ. The metabolism of asparagine in plants. Phytochemistry 1988; 27: 663-671.

Simoh S, Linthorst HJ, Lefeber AW, Erkelens C, Kim HK, Choi YH, Verpoorte R. 630 Metabolic changes of Brassica rapa transformed with a bacterial isochorismate synthase gene. Journal of Plant Physiology 2010; 167: 1525-1532.

Simoh S, Quintana N, Kim HK, Choi YH, Verpoorte R. Metabolic changes in Agrobacterium tumefaciens-infected Brassica rapa. Journal of Plant Physiology 2009; 166: 1005-1014.

635 Sticher L, MauchMani B, Metraux JP. Systemic acquired resistance. Annual Review of Phytopathology 1997; 35: 235-270.

Suzuki T, Honda Y, Mukasa Y. Effects of UV-B radiation, cold and desiccation stress on rutin concentration and rutin glucosidase activity in tartary buckwheat (Fagopyrum tataricum) leaves. Plant Science 2005; 168: 1303-1307.

640 Tan JW, Bednarek P, Liu HK, Schneider B, Svatos A, Hahlbrock K. Universally occurring phenylpropanoid and species-specific indolic metabolites in infected and uninfected Arabidopsis thaliana roots and leaves. Phytochemistry 2004; 65: 691-699.

Urbanczyk-Wochniak E, Baxter C, Kolbe A, Kopka J, Sweetlove LJ, Fernie AR. Profiling of diurnal patterns of metabolite and transcript abundance in potato (Solanum 645 tuberosum) leaves. Planta 2005; 221: 891-903.

van Kan JAL, Joosten MHAJ, Wagemakers CAM, Vandenbergvelthuis GCM, Dewit PJGM. Differential accumulation of messenger-Rnas encoding extracellular and intracellular Pr proteins in tomato induced by virulent and avirulent races of Cladosporium fulvum. Plant Mol Biol 1992; 20: 513-527.

650 van Loon LC, Rep M, Pieterse CMJ. Significance of inducible defense-related proteins in infected plants. Annu Rev Phytopathol 2006; 44: 135-162.

Verpoorte R, Choi YH, Kim HK. NMR-based metabolomics at work in phytochemistry. Phytochemistry Reviews 2007; 6: 3-14.

Verpoorte R, Choi HY, Mustafa NR, Kim HK. Metabolomics: back to basics. 655 Phytochem Rev 2008; 7: 525-537.

Vlot A, Dempsey DA, Klessig DF. Salicylic acid, a multifaceted hormone to combat disease. 2009, 177-206 pp.

Wallace W, Secor J, Schrader LE. Rapid accumulation of Gamma-aminobutyric acid and alanine in soybean leaves in response to an abrupt transfer to lower temperature, 660 darkness, or mechanical manipulation. Plant Physiology 1984; 75: 170-175.

Ward JL, Forcat S, Beckmann M, Bennett M, Miller SJ, Baker JM, Hawkins ND, Vermeer CP, Lu C, Lin W, Truman WM, Beale MH, Draper J, Mansfield JW, Grant M. The metabolic transition during disease following infection of Arabidopsis thaliana by Pseudomonas syringae pv. tomato. Plant Journal 2010; 63: 443-457. 
665 Wolfender JL. HPLC in natural product analysis: the detection issue. Planta Medica 2009; 75: 719-734.

Zacarés L, López-Gresa MP, Fayos J, Primo J, Bellés JM, Conejero V. Induction of $p$ coumaroyldopamine and feruloyldopamine, two novel metabolites, in tomato by the bacterial pathogen Pseudomonas syringae. Molecular Plant-Microbe Interactions 2007;

670 20: 1439-1448. 


\section{Figures}

Figure 1. SDS-PAGE analysis of soluble proteins in extracts from Tomato Mosaic

675 Virus (ToMV)-inoculated and the corresponding upper systemically infected leaves at the indicated time (days) post-inoculation. Protein size markers $(\mathrm{kDa})$ are indicated on the left. Arrow on the right indicates the ToMV coat protein.

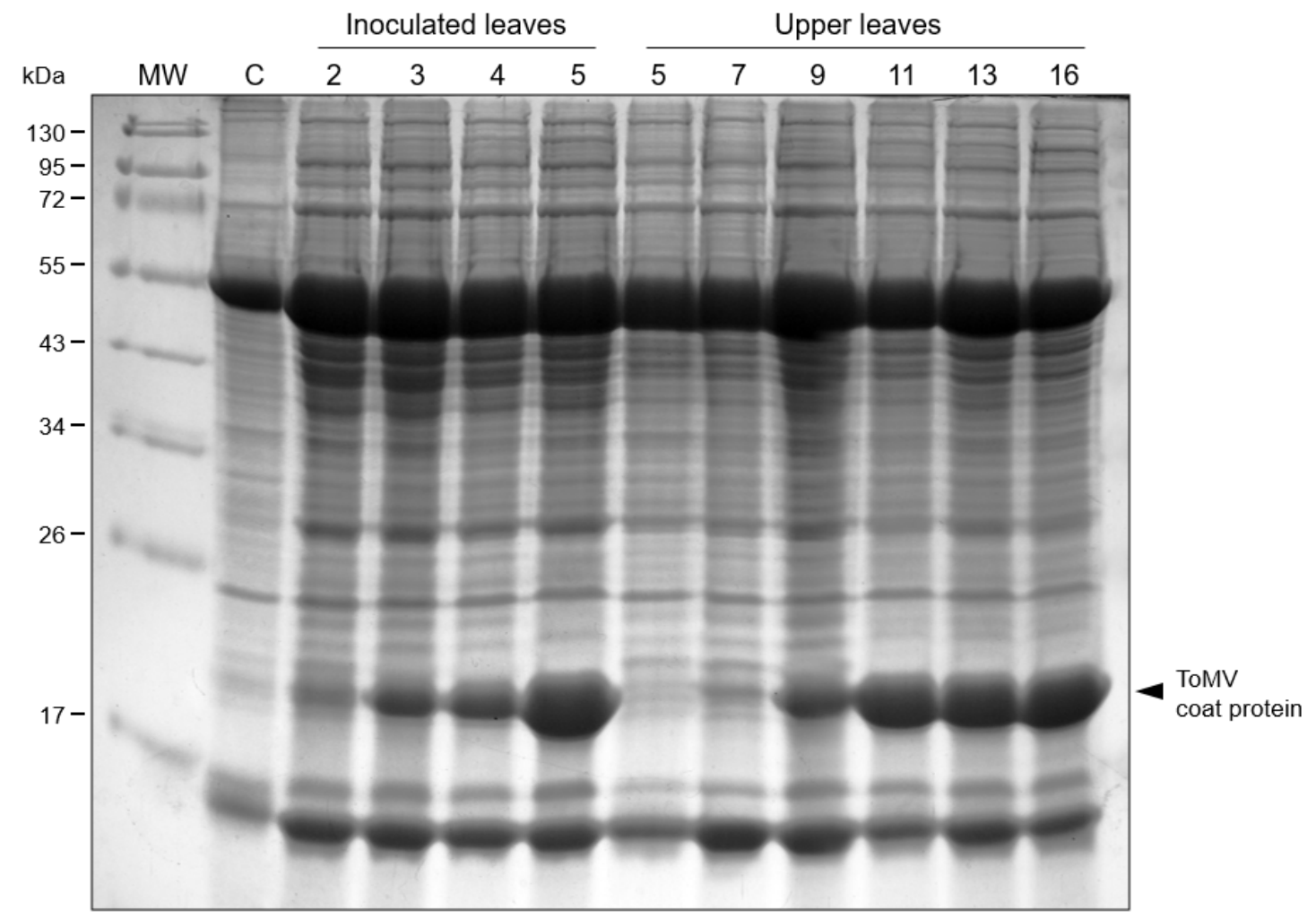


680 Figure 2. Score plot of PLS based on the whole range of the ${ }^{1} \mathrm{H}$ NMR signals in the range of $\delta$ 0.3-10.0. The numbers indicate the days after mock and ToMV inoculation.

(x) lower leaves of mock-and ToMV-inoculated tomato plants

(•) upper leaves of healthy control and ToMV-infected tomato plants.

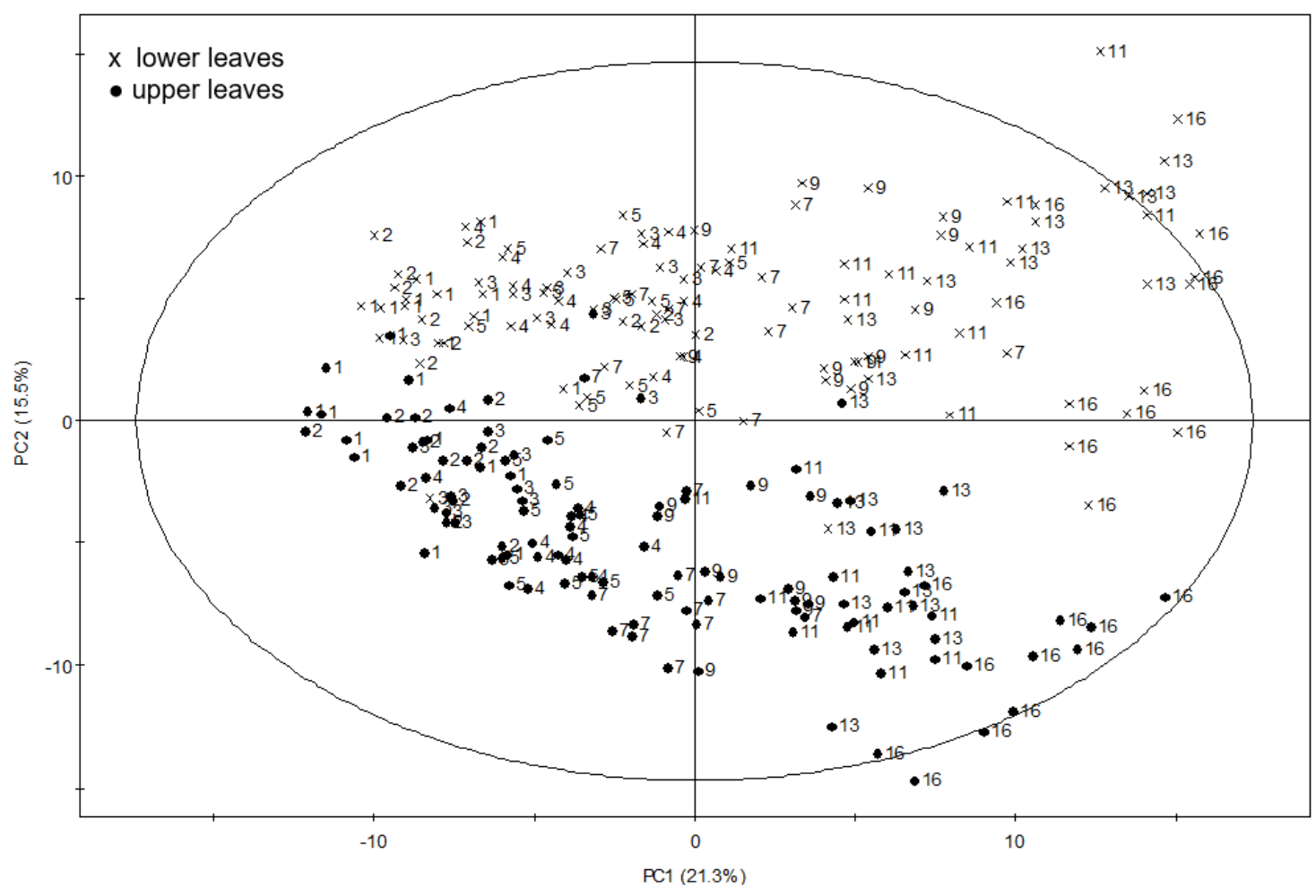


Figure 3. Score plot of PLS based on the whole range of the ${ }^{1} \mathrm{H}$ NMR signals in the range of $\delta$ 0.3-10.0. The numbers indicate the days after mock and ToMV inoculation.

(o) lower mock-inoculated and upper leaves of healthy tomato plants

(घ) lower ToMV-inoculated and upper systemically-infected leaves of tomato plants.

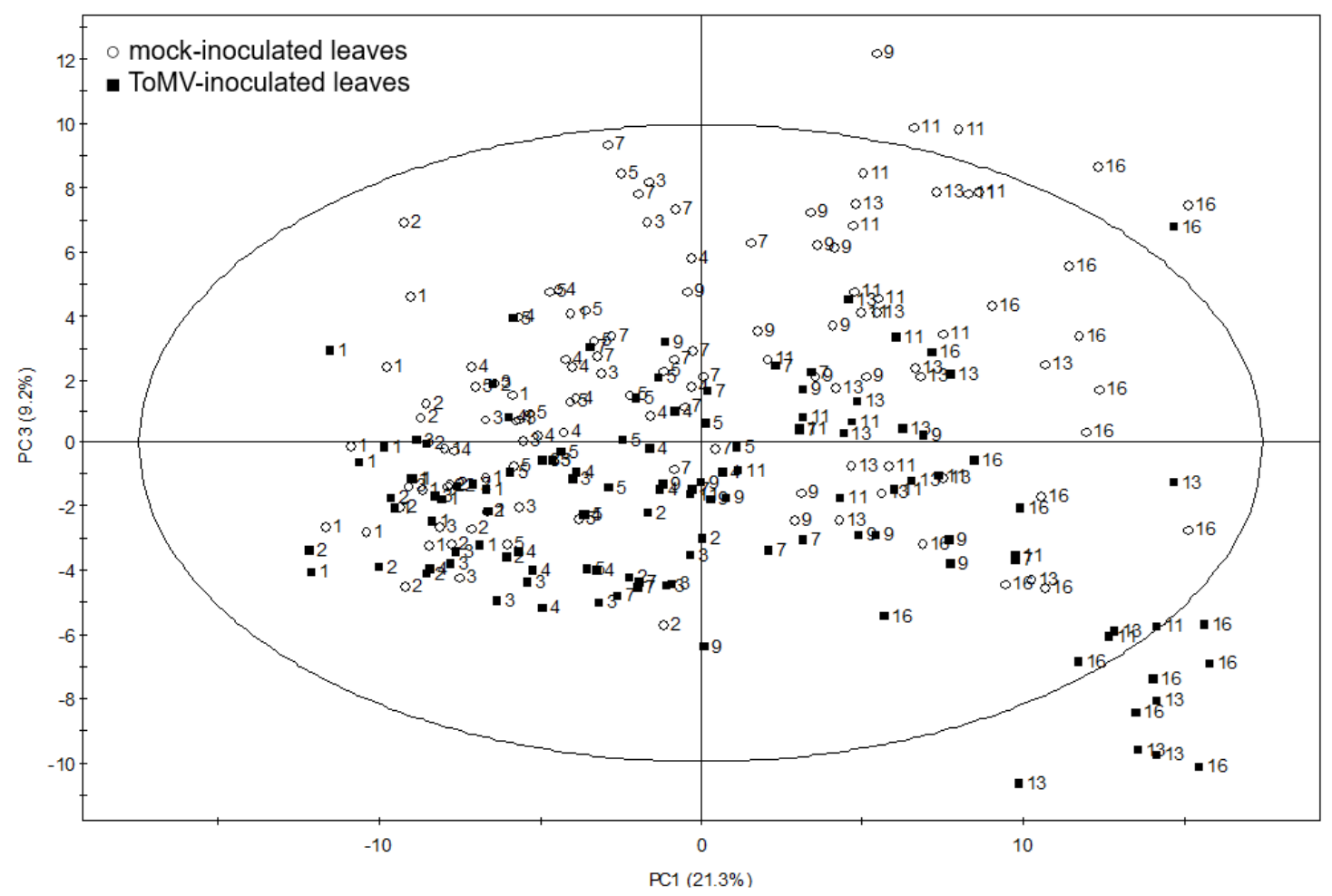


Figure 4. Score plot of PLS based on the whole range of the ${ }^{1} \mathrm{H}$ NMR signals in the range of $\delta$ 0.3-10.0. The numbers indicate the days after mock and ToMV inoculation.

( $\boldsymbol{\Delta}$ ) leaves harvested at 9:00 h from mock-inoculated and ToMV-infected tomato 695 plants. $(\Delta)$ leaves harvested at 19:00 h from mock-inoculated and ToMV-infected tomato plants.

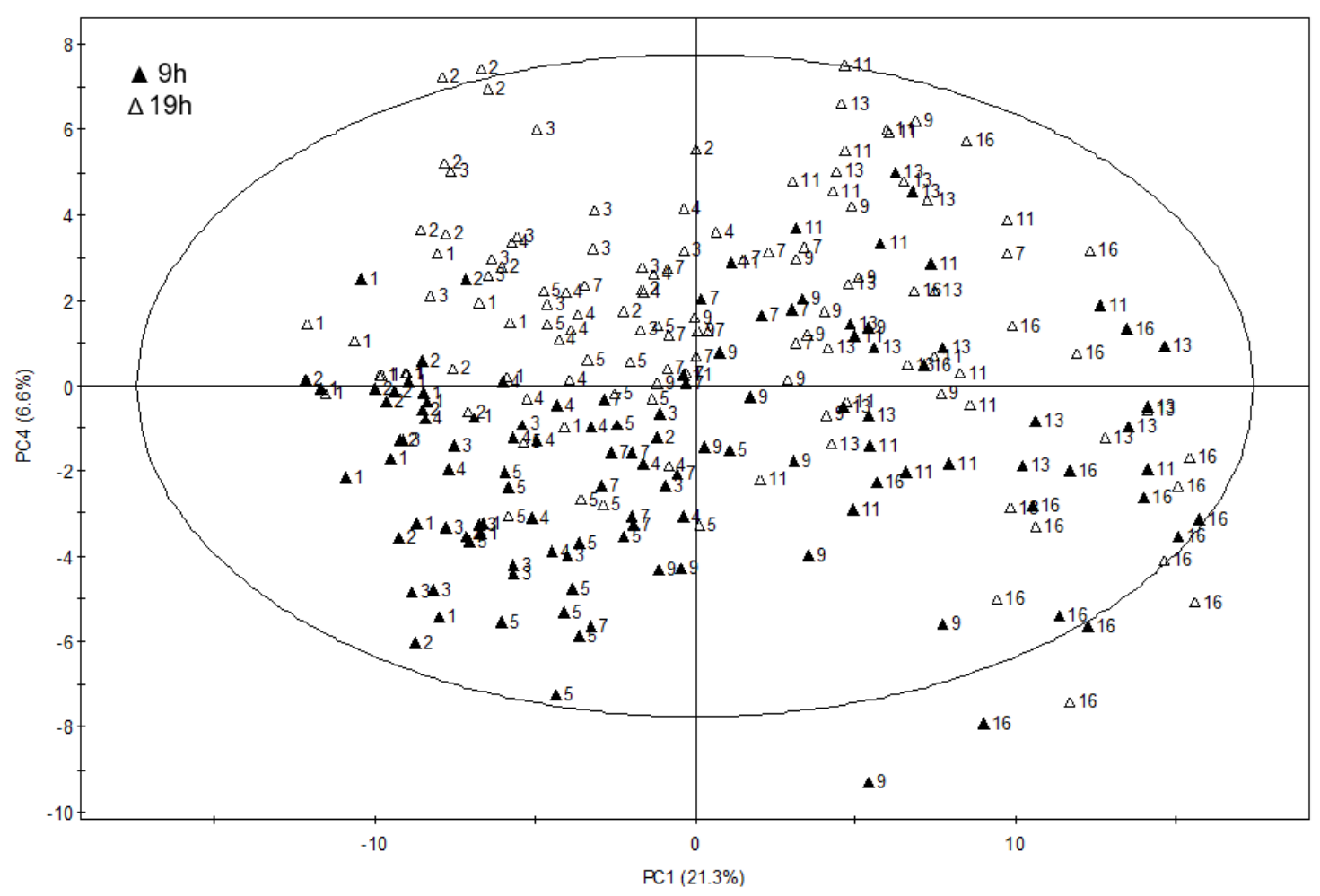


Figure 5A. PLS-DA score plot of early local viral infection based on the ${ }^{1} \mathrm{H}$ NMR signals in the range of $\delta$ 0.3-10.0. The numbers indicate the days after mock and ToMV inoculation.

(०) mock-inoculated leaves of healthy tomato plants

(a) ToMV-inoculated leaves of infected tomato plants.

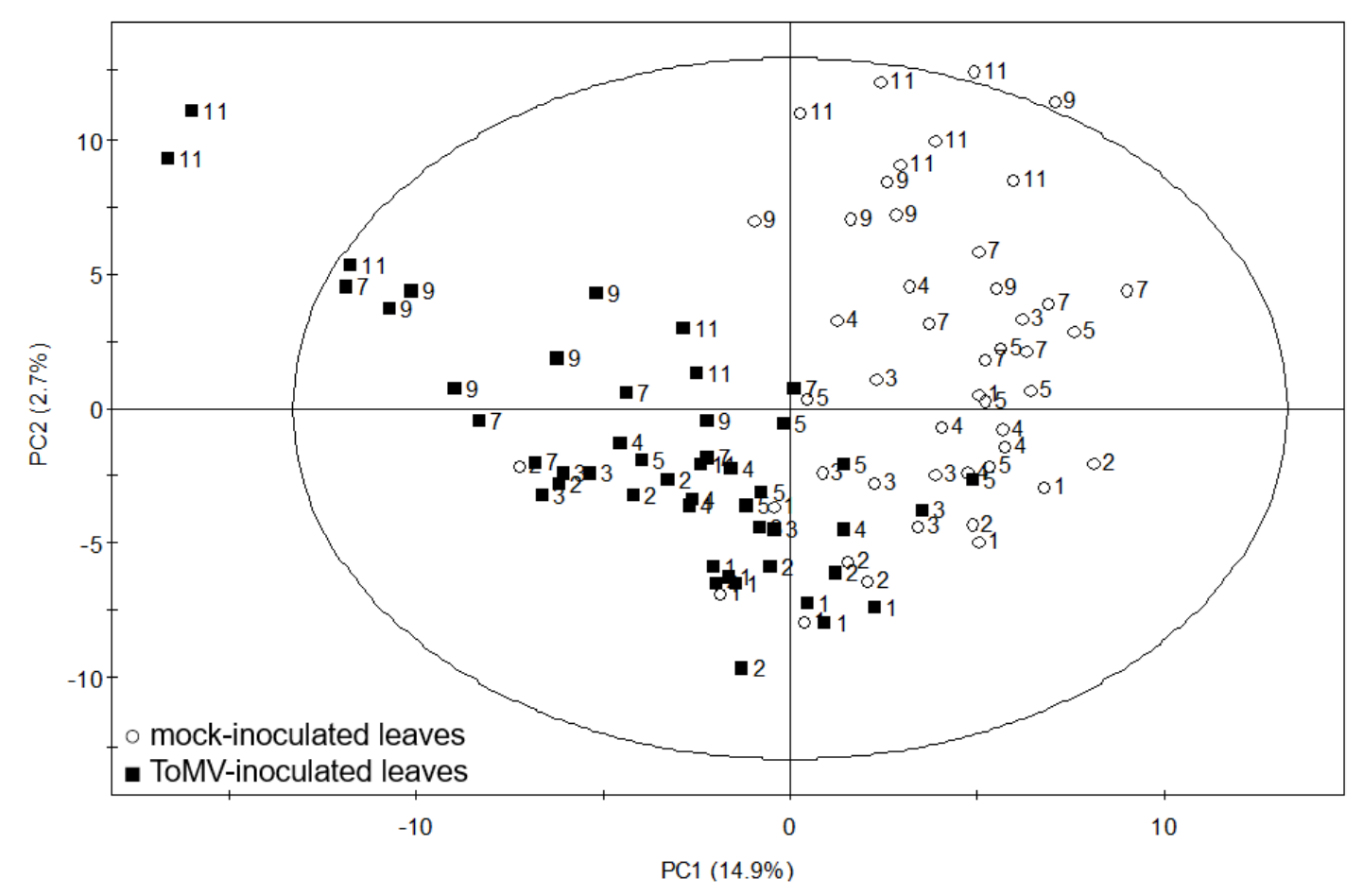


Figure 5B. PLS-DA score plot of delayed systemic viral infection on the ${ }^{1} \mathrm{H}$ NMR signals in the range of $\delta$ 0.3-10.0. The numbers indicate the days after mock and ToMV inoculation.

( $)$ upper leaves of healthy tomato plants

710 (घ) systemically ToMV-infected leaves of inoculated tomato plants.

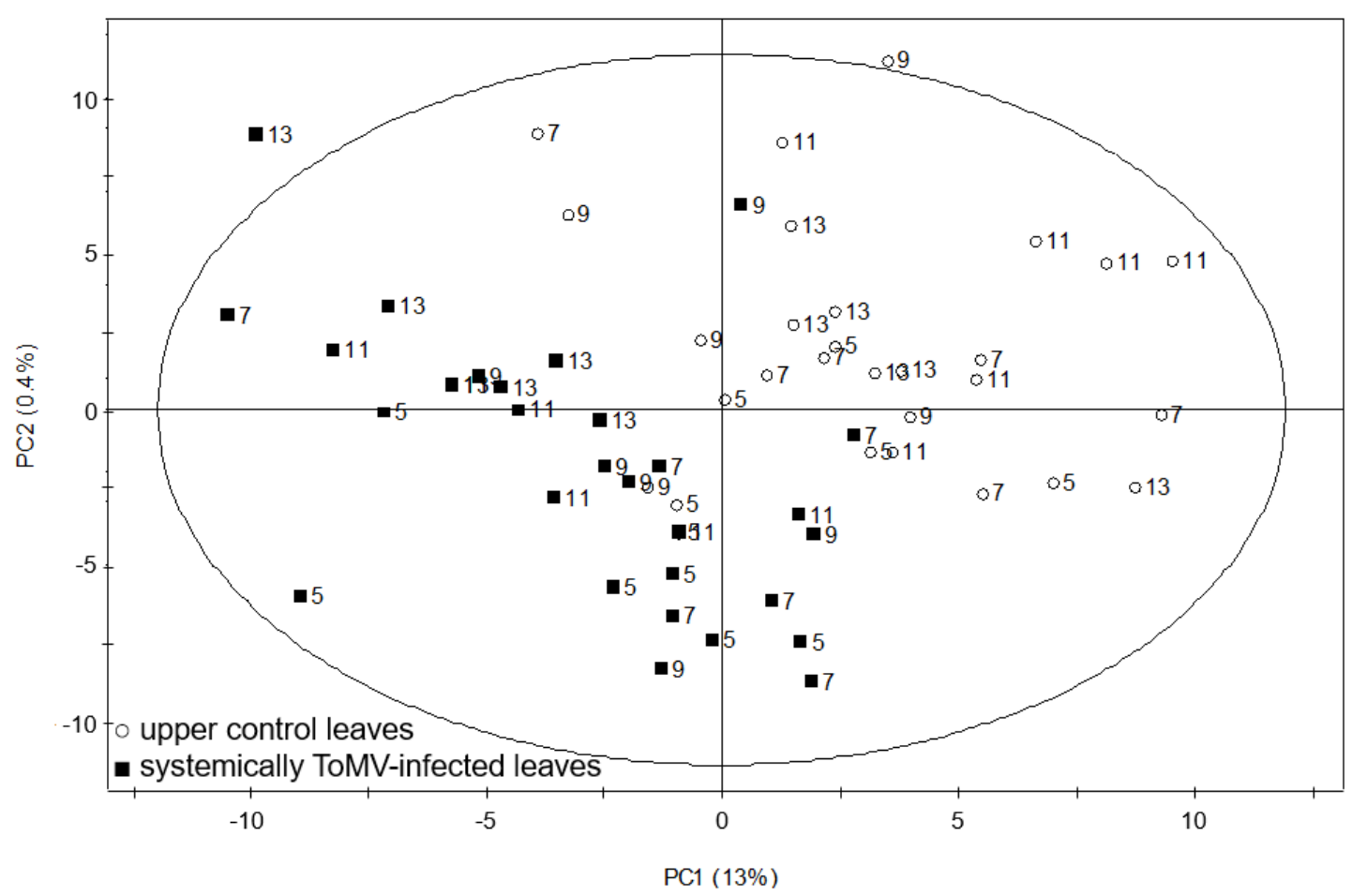


Figure 6. Column loading plot of PLS-DA component 1 on upper healthy and ToMV systemically infected leaves.

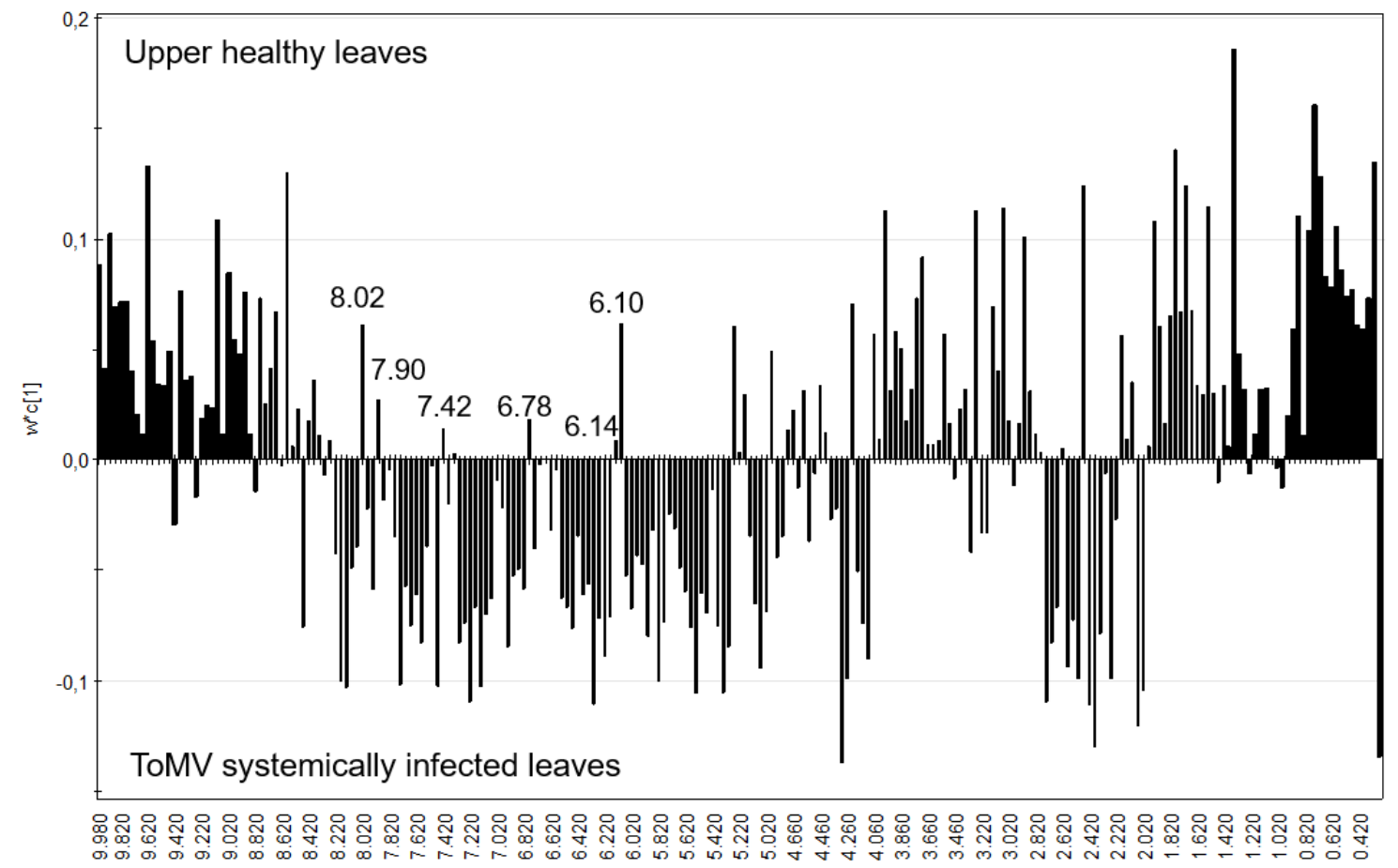

NMR chemical shifts (ppm) 
Table 1. Compounds identified from $1 \mathrm{H}-\mathrm{NMR}$ and 2D NMR spectra in $50 \% \mathrm{CH}_{3} \mathrm{OH}-\mathrm{d}_{4}$ in $\mathrm{D}_{2} \mathrm{O}\left(\mathrm{KH}_{2} \mathrm{PO}_{4}\right.$ buffer $\mathrm{pH}$ 6) of control and ToMV infected S.lycopersicum leaves.

720 ( $\uparrow$ ): increase, $(\downarrow)$ decrease, (=) no change, (L): local infection. (S): systemic infection

\begin{tabular}{|c|c|c|c|c|c|c|}
\hline \multirow[t]{2}{*}{ Metabolites } & \multirow{2}{*}{$\begin{array}{c}\text { Chemical shift ( } \delta \text { in ppm) and } \\
\text { coupling constant ( } \mathrm{J} \text { in Hz) }\end{array}$} & \multirow{2}{*}{$\begin{array}{l}\text { Age } \\
\text { (old) }\end{array}$} & \multirow{2}{*}{$\begin{array}{l}\text { Development } \\
\text { stage (distal) }\end{array}$} & \multicolumn{2}{|c|}{ Infection } & \multirow{2}{*}{$\begin{array}{l}\text { Collection } \\
\text { time (19h) }\end{array}$} \\
\hline & & & & $\mathrm{L}$ & S & \\
\hline \multicolumn{7}{|c|}{ Amino/organic acids } \\
\hline Isoleucine & $\begin{array}{l}0.95(\mathrm{t}, 7.5), 1.02(d, 7.0), 1.26(\mathrm{~m}), \\
1.50(\mathrm{~m}), 1.98(\mathrm{~m}), 3.61(\mathrm{~d}, 4.0)\end{array}$ & $\downarrow$ & $=$ & $\downarrow$ & $=$ & $\downarrow$ \\
\hline Leucine & $0.97(d, 6.4), 0.98(d, 6.4), 1.72(m)$ & $\downarrow$ & $=$ & $\downarrow$ & $=$ & $\downarrow$ \\
\hline Valine & $\begin{array}{l}1.00(d, 7.2), 1.05(d, 7.2), 2.28(m), \\
3.54(d, 4.0)\end{array}$ & $\downarrow$ & $\uparrow$ & $\downarrow$ & $=$ & $\downarrow$ \\
\hline Threonine & $1.33(d, 6.6), 3.53(d, 5.0), 4.23(\mathrm{~m})$ & $=$ & $=$ & $\downarrow$ & $\downarrow$ & $\downarrow$ \\
\hline Alanine & $1.48(d, 7.2), 3.73(\mathrm{~m})$ & $\downarrow$ & $\uparrow$ & $\downarrow$ & $\downarrow$ & $\downarrow$ \\
\hline Arginine & $\begin{array}{l}1.67(\mathrm{~m}), 1.72(\mathrm{~m}), 3.24(\mathrm{~m}), 3.67(\mathrm{t}, \\
5.8)\end{array}$ & $\downarrow$ & $\uparrow$ & $\downarrow$ & $\downarrow$ & $\downarrow$ \\
\hline GABA & $1.90(\mathrm{~m}), 2.31(t, 7.2), 3.01(t, 7.2)$ & $=$ & $\downarrow$ & $\uparrow$ & $\uparrow$ & $\downarrow$ \\
\hline Acetic acid & $1.94(s)$ & $\uparrow$ & $\uparrow$ & $\downarrow$ & $\downarrow$ & $\downarrow$ \\
\hline Glutamic acid & $\begin{array}{l}2.05(m), 2.13(m), 2.39(t d, 7.2,2.4), \\
3.70(d d, 7.2,4.3)\end{array}$ & $\downarrow$ & $\downarrow$ & $\uparrow$ & $\uparrow$ & $\uparrow$ \\
\hline Glutamine & $2.14(\mathrm{~m}), 2.45(\mathrm{~m}), 3.71(\mathrm{~m})$ & $\downarrow$ & $\downarrow$ & $\uparrow$ & $\downarrow$ & $\downarrow$ \\
\hline Malic acid & $\begin{array}{l}2.47(d d, 15.8,8.3), 2.72(d d, 15.8, \\
3.9), 4.29(d d, 8.3,3.9)\end{array}$ & $\downarrow$ & $\downarrow$ & $\uparrow$ & $\uparrow$ & $\downarrow$ \\
\hline Citric acid & $2.53(d, 16.3), 2.71(d, 16.3)$ & $\downarrow$ & $\downarrow$ & $\uparrow$ & $\uparrow$ & $\downarrow$ \\
\hline Aspartic acid & $\begin{array}{l}2.65(d d, 17.3,8.8), 2.81(d d, 17.3 \\
4.0), 3.84(d d, 8.8,4.0)\end{array}$ & $\downarrow$ & $\downarrow$ & $\uparrow$ & $\uparrow$ & $\downarrow$ \\
\hline Asparagine & $\begin{array}{l}2.81(d d, 17.0,8.2), 2.95(d d, 17.0, \\
4.0), 3.94(d d, 8.2,4.0)\end{array}$ & $\uparrow$ & $\downarrow$ & $\uparrow$ & $\downarrow$ & $\downarrow$ \\
\hline Tryptophan & $\begin{array}{l}7.15(t d, 7.9,2.0), 7.24(t d, 7.9,2.0), \\
7.29(s), 7.49(d, 8.0), 7.71(d, 7.9)\end{array}$ & $=$ & $\downarrow$ & $\downarrow$ & $\uparrow$ & $\downarrow$ \\
\hline Fumaric acid & $6.54(s)$ & $\downarrow$ & $\downarrow$ & $\uparrow$ & $\uparrow$ & $=$ \\
\hline Phenylalanine & $7.35(m)$ & $\downarrow$ & $\downarrow$ & $\uparrow$ & $\uparrow$ & $\downarrow$ \\
\hline Formic acid & $8.46(s)$ & $\uparrow$ & $\downarrow$ & $\uparrow$ & $\uparrow$ & $\downarrow$ \\
\hline Sugars & & & & & & \\
\hline
\end{tabular}




\begin{tabular}{|c|c|c|c|c|c|c|}
\hline Sucrose & $4.17(d, 8.6), 5.41(d, 3.8)$ & $\uparrow$ & $\uparrow$ & $\downarrow$ & $\uparrow$ & $\uparrow$ \\
\hline Glucose ( $\alpha, \beta$ form) & $5.18(d .3 .8), 4.58(d, 7.9)$ & $\uparrow$ & $\uparrow$ & $\uparrow$ & $\downarrow$ & $\uparrow$ \\
\hline \multicolumn{7}{|l|}{$\begin{array}{l}\text { Phenylpropanoids/ } \\
\text { Flavonoids }\end{array}$} \\
\hline $\begin{array}{l}\text { Caffoyl esters of } \\
\text { polyhydroxy } \\
\text { compounds (glucaric } \\
\text { acid) (4) }\end{array}$ & $\begin{array}{l}5.10(d, 4.9), 6.47(d, 16.0), 6.98(d, \\
8.2), 7.06(d d, 8.2,2.0), 7.18(d, 2.0), \\
7.66(d, 16.0)\end{array}$ & $\downarrow$ & $\downarrow$ & $\downarrow$ & $\uparrow$ & $\uparrow$ \\
\hline Rutin & $\begin{array}{l}6.33(d, 2.1), 6.54(d, 2.1), 6.99(d, \\
8.4), 5.02(d, 7.8)\end{array}$ & $\downarrow$ & $\downarrow$ & $\uparrow$ & $\uparrow$ & $\uparrow$ \\
\hline \multicolumn{7}{|l|}{ Other compounds } \\
\hline Dimethylamine & $2.97(s)$ & $\uparrow$ & $\uparrow$ & $\uparrow$ & $\uparrow$ & $\uparrow$ \\
\hline Ethanolamine & $3.12(t, 5.5)$ & $=$ & $=$ & $=$ & $\downarrow$ & $\downarrow$ \\
\hline Choline & $3.21(s)$ & $\uparrow$ & $\uparrow$ & $\uparrow$ & $\uparrow$ & $\downarrow$ \\
\hline Trigonelline & $\begin{array}{l}4.46(s), 8.10(d d, 1.8,6.2), 8.87(m), \\
9.14(s)\end{array}$ & $=$ & $\uparrow$ & $=$ & $\downarrow$ & $\downarrow$ \\
\hline UDP-Glucose & $5.96(d, 8.1), 7.97(d, 8.1)$ & $\uparrow$ & $=$ & $\downarrow$ & $\uparrow$ & $\uparrow$ \\
\hline Adenosin & $5.96(d,, 4.4), 8.24(s), 8.54(s)$ & $\downarrow$ & $\downarrow$ & $\downarrow$ & $=$ & $\downarrow$ \\
\hline
\end{tabular}

